\title{
Analog Beamformer Design for Interference Exploitation based Hybrid Beamforming
}

\author{
Ganapati Hegde*, Christos Masouros ${ }^{\dagger}$, Marius Pesavento* \\ ${ }^{*}$ Communication Systems Group, Technische Universität Darmstadt, Germany \\ ${ }^{\dagger}$ Department of Electrical and Electronic Engineering, University College London, UK
}

\begin{abstract}
Hybrid analog-digital beamforming has been shown to reduce hardware cost and power consumption in massive MIMO systems, at the expense of increased radiated power for given performance targets. To alleviate the above shortfall, in this paper we exploit the concept of constructive interference (CI) that has been shown to offer significant radiated power savings in fully-digital multi-user downlink MIMO systems. We explore analog beamforming design, and develop solutions specifically tailored for CI-based hybrid beamforming. We also evaluate the performance of the presented techniques and their computational complexities using simulation results.
\end{abstract}

\section{INTRODUCTION}

The hybrid analog-digital beamforming [1-6] has become popular in recent years as a judicious approach of making massive MIMO system practical and economical for the upcoming 5G networks [7, 8]. Unlike the conventional fullydigital beamforming (fully-DBF) [9, 10], which requires a dedicated radio frequency $(\mathrm{RF})$ chain for each antenna, the hybrid beamforming can be realized using a fewer number of RF chains compared to the number of antennas. Even though hybrid beamforming substantially reduces the hardware cost and the power consumption associated with the RF electronic circuits, inadvertently it increases the transmitted (radiated) power to achieve the same performance as that of the conventional fully-DBF. Therefore, techniques to improve the power efficiency are even more critical in hybrid beamforming based wireless networks than in the conventional networks. One such approach to improve the power efficiency in the system is the constructive interference (CI) technique [1115]. In the CI technique, the signals produced by all transmit beamformers are aligned at the user terminals such that the resultant signals lie in the desired region of the corresponding transit symbols in the constellation diagram (see Section III for the precise definition of the desired region).

In general, optimal design of analog and digital beamformers in hybrid beamforming is a non-convex problem and difficult to solve. A common approach to design hybrid beamformers is to decompose the problem into two parts, where the analog beamformers (ABFs) are designed in the first part and then, for the fixed ABFs, the optimal digital beamformers (DBFs) are designed $[2-4,16]$. In this paper, our objective is to evaluate the performance of different $\mathrm{ABF}$ design techniques in a CI based hybrid beamforming system. We consider both continuous-valued and codebook-based ABF design techniques. For the codebook-based ABF design, we study two types of codebooks, namely, the discrete Fourier

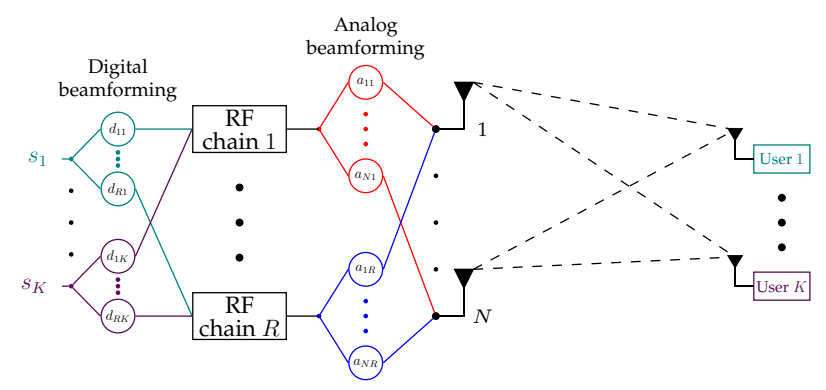

Fig. 1: Schematic diagram of hybrid beamforming system.

transform (DFT) matrix based codebook and the random phase constant modulus codebook. Once the ABFs are fixed, the optimal DBFs for the corresponding ABFs are obtained to compute the total transmitted power, which facilitate a fair comparison among ABF design techniques. Simulation results are presented to compare the performance of various $\mathrm{ABF}$ design techniques in terms of total transmit power and computation time. We also evaluate the effect of employed codebook type on the performance of the presented codebookbased ABF design techniques.

\section{SySTEM MODEL}

Consider a co-channel multi-user downlink massive MIMO system with a base station (BS) equipped with $N$ transmit antennas and $R$ RF chains, where $R \ll N$, as shown in Fig. 1. Let $\mathcal{K} \triangleq\{1, \ldots, K\}$ denote a set of $K$ single antenna users present in the system. Let $\mathbf{s}=\left[s_{1}, \ldots, s_{K}\right]^{\top}$ be the transmit symbol vector at the BS, where the element $s_{k}$ denotes the symbol intended for the $k$ th user. Each symbol is assumed to be drawn from an $M$-ary phase-shift keying ( $M$ PSK) constellation. Without loss of generality, each symbol is assumed to be unit modulus, i.e., $s_{k}=\exp \left(j \phi_{k}\right)$ for $\phi_{k} \in\left\{\Phi_{1}, \ldots, \Phi_{M}\right\}$, where $0 \leq \Phi_{m} \leq 2 \pi$. A DBF $\mathbf{d}_{k} \in \mathbb{C}^{R}$ is applied to the transmit symbol $s_{k}$ and the resulting signals are fed to the $R \mathrm{RF}$ chains, for $k \in \mathcal{K}$. Each $\mathrm{RF}$ chain is connected to all transmit antennas through analog phase shifters (PSs), having a constant magnitude $a$. Let $a_{n r}$ denote the value of the PS that connects the $n$th antenna to the $r$ th RF chain, with a phase value $\rho_{n r}$, i.e., $a_{n r}=a \exp \left(j \rho_{n r}\right)$ for $n \in \mathcal{N}, r \in \mathcal{R} \triangleq\{1, \ldots, R\}$. Then $\mathbf{a}_{r}=\left[a_{1 r}, \ldots, a_{N r}\right]^{\top}$ forms the ABF applied to the output of the $r$ th RF chain. The matrix $\mathbf{A} \triangleq\left[\mathbf{a}_{1}, \ldots, \mathbf{a}_{R}\right]$ represents the ABF matrix.

Let $\tilde{\mathbf{h}}_{k} \in \mathbb{C}^{N}$ be the vector characterizing the frequencyflat channel between the $\mathrm{BS}$ and the $k$ th user, and $\tilde{\mathbf{H}}=$ 


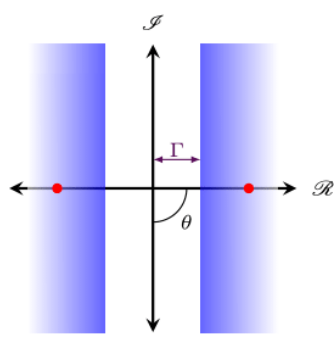

(a) BPSK

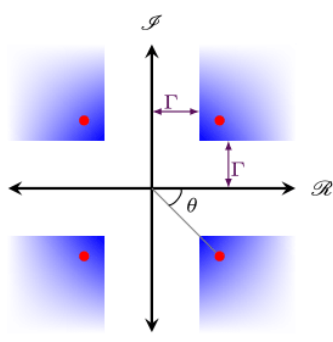

(b) QPSK

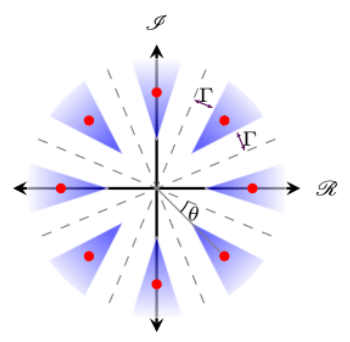

(c) 8-PSK

Fig. 2: The desired region (in shaded blue) of the constellation points for different modulation schemes.

$$
\begin{aligned}
\underset{\substack{\left.\mathbf{d}_{k}\right\}_{k \in \mathcal{K}},\left\{a_{n r}\right\}_{r \in \mathcal{R}}^{n \in \mathcal{N}} \\
\operatorname{minimize}}}{\text { s.t. }} & \left|\operatorname{Im}\left(\tilde{\mathbf{h}}_{k}^{\top} \mathbf{A} \sum_{i=1}^{K} \mathbf{d}_{i} s_{i}\right)\right| \leq\left(\operatorname{Re}\left(\tilde{\mathbf{h}}_{i}^{\top} \mathbf{A} \sum_{i=1}^{K} \mathbf{d}_{i} s_{i}\right)-\gamma_{k}\right) \tan \theta, \quad \forall k \in \mathcal{K}, \\
& \left|a_{n r}\right|=a, \quad \forall n \in \mathcal{N}, \forall r \in \mathcal{R} .
\end{aligned}
$$

$\left[\tilde{\mathbf{h}}_{1}, \ldots, \tilde{\mathbf{h}}_{K}\right]$ denote the corresponding channel matrix. The complete channel state information $\tilde{\mathbf{H}}$ is assumed to be known at the BS $[17,18]$. The received signal $y_{k}$ at the $k$ th user can be expressed as

$$
y_{k}=\sum_{i=1}^{K} \tilde{\mathbf{h}}_{k}^{\top} \mathbf{A d}_{i} s_{i}+n_{k}
$$

where $n_{k}$ denotes the i.i.d. complex additive white Gaussian noise at the $k$ th user, with zero mean and variance $\sigma_{k}^{2}$.

\section{Problem Formulation}

As depicted in Fig. 2, we define the desired region (in shaded blue) of a constellation point as the (continuous) set of points in the complex domain that are, a) closer to the corresponding constellation point than any other constellation points, and b) at least $\Gamma$ distance apart from any of the decision boundaries of the corresponding constellation point [12, 19]. The parameter $\Gamma$ is called as the threshold-margin. Note that, while Fig. 2 depicts examples of PSK modulations, the above concept also applies to generic constellation formats [20]. Let $\theta=\pi / M$ represent the angle between any constellation point and decision boundaries of the corresponding constellation point. Now, the problem of designing joint analogdigital beamformers, enforcing the $\mathrm{CI}$ at each user, can be formulated as a non-convex optimization problem (1), where $\gamma_{k}=\Gamma_{k} / \sin \theta$ with $\Gamma_{k}$ indicating the threshold-margin of the $k$ th user. In this problem the objective function (1a) minimizes the total transmit power, and the constraints in (1b) enforce the received signal at the $k$ th user to lie in the desired region of the transmit symbol of the $k$ th user. We remark that the thresholdmargin of the $k$ th user $\Gamma_{k}$ in the above problem influences the symbol detection error rate, and hence determines the quality of service experienced by the $k$ th user.
Following a process similar to the one in [12] for the DBF design, the problem (1) can be reformulated as an equivalent single-group multicast problem given by,

$$
\begin{aligned}
\underset{\mathbf{b},\left\{a_{n r}\right\}_{r \in \mathcal{R}}^{n \in \mathcal{N}}}{\operatorname{minimize}} & \mid \mathbf{A} \mathbf{b} \|^{2} \\
\text { s.t. } & \left|\operatorname{Im}\left(\mathbf{h}_{k}^{\top} \mathbf{A} \mathbf{b}\right)\right| \leq\left(\operatorname{Re}\left(\mathbf{h}_{k}^{\top} \mathbf{A} \mathbf{b}\right)-\gamma_{k}\right) \tan \theta, \quad \forall k \in \mathcal{K}, \\
& \left|a_{n r}\right|=a, \quad \forall n \in \mathcal{N}, \forall r \in \mathcal{R}, \quad
\end{aligned}
$$

where the modified channel of the $k$ th user $\mathbf{h}_{k}$ is given by, $\mathbf{h}_{k} \triangleq \tilde{\mathbf{h}}_{k} \exp \left(j\left(\phi_{1}-\phi_{k}\right)\right)$. Note that, the transmit symbol $s_{k}$ is now incorporated into the modified channel $\mathbf{h}_{k}$. Consequently, the values of modified channel $\mathbf{h}_{k}$ vary at every symbol interval. The optimal DBF $\mathbf{b}^{\star}$ of problem (3) and the optimal DBFs $\mathbf{d}_{k}^{\star}$ of problem (1) are related as

$$
\mathbf{d}_{k}^{\star}=\frac{\mathbf{b}^{\star}}{K} \exp \left(j\left(\phi_{1}-\phi_{k}\right)\right), \quad \forall k \in \mathcal{K} .
$$

The above joint analog-digital beamforming problem is a nonconvex problem due to a) non-convex domain of the $\mathrm{ABF}$ elements $\left.a_{n r}, \mathrm{~b}\right)$ the bi-linear coupling of the ABF matrix $\mathbf{A}$ and the DBF $\mathbf{b}$, and hard to solve optimally. Therefore, we adopt the successive optimization technique, and decompose the problem into two parts. Firstly, we design the ABF matrix, and then for the fixed ABF matrix $\hat{\mathbf{A}}$, the optimal $\mathrm{DBF} \mathbf{b}^{\star}$ is designed by solving following convex problem [12]:

$$
\begin{aligned}
& \underset{\mathbf{b}}{\operatorname{minimize}}\|\hat{\mathbf{A}} \mathbf{b}\|^{2} \\
& \text { s.t. }\left|\operatorname{Im}\left(\mathbf{h}_{k}^{\top} \hat{\mathbf{A}} \mathbf{b}\right)\right| \leq\left(\operatorname{Re}\left(\mathbf{h}_{k}^{\top} \hat{\mathbf{A}} \mathbf{b}\right)-\gamma_{k}\right) \tan \theta, \\
& \forall k \in \mathcal{K} .
\end{aligned}
$$

In this paper our focus is to study different $A B F$ matrix design techniques, and compare their performances and computational complexities. 


\section{Analog BeAmformer Design Techniques}

Analog beamforming is employed in the RF domain on the output of the RF chains. As ABFs are realized using analog PSs, their elements have constant magnitude and differ only in their phase values. There are two types of ABFs that are generally used in hybrid beamforming systems, namely, continuous-valued ABFs and codebook-based ABFs [16, 2123]. A continuous-valued $\mathrm{ABF}$ provides more degrees of freedom, as each of its elements can take any phase value between 0 and $2 \pi$. However, its realization requires expensive high resolution tunable phase shifters. On the other hand, in a codebook-based analog beamforming system, the ABFs are selected from a predefined codebook that is commonly realized in hardware with switchable spatial filter banks composed of inexpensive fixed phase shifters.

\section{A. Continuous-valued ABF design}

Conjugate Phase of Channel (CPC) method: When the number of RF chains $R$ is same as the number of users $K$, a dedicated RF chain can be assigned to each user. Then the array gain between the $k$ th user and a corresponding RF chain can be maximized by assigning the conjugate phase values of the channel vector $\tilde{\mathbf{h}}_{k}$ to the corresponding ABF $\mathbf{a}_{k}$ [24]. Consequently, the $(n, k)$ th element of the ABF matrix $\mathbf{A}$ can be expressed as,

$$
A_{n, k}=\frac{1}{\sqrt{N}} e^{-j \beta_{n, k}}, \quad \forall n \in \mathcal{N}, \forall k \in \mathcal{K},
$$

where $\beta_{n, k}$ indicates the phase value of $n$th element of channel vector $\tilde{\mathbf{h}}_{k}$. We remark that in this method the ABF matrix is designed based on the channel vector $\tilde{\mathbf{h}_{k}}$, and hence it can be kept fixed as long as the channel vector remains the same.

\section{B. Codebook-based ABF design}

In the codebook based ABF design techniques, the ABFs are chosen from a predefined set $\mathcal{C}$ comprising $L$ normalized ABFs, where $L \geq R$. Let $\mathbf{c}_{\ell} \in \mathbb{C}^{N}$ denote the $\ell$ th element of set $\mathcal{C}$, and $\mathbf{C}=\left[\mathbf{c}_{1}, \ldots, \mathbf{c}_{L}\right] \in \mathbb{C}^{N \times L}$ be the codebook matrix. All the elements of the codebook matrix $\mathbf{C}$ have a constant magnitude $1 / \sqrt{N}$, so that $\left\|\mathbf{c}_{\ell}\right\|=1$. In the following, we discuss different techniques to choose $R$ ABFs, one for each $\mathrm{RF}$ chain, from the codebook $\mathcal{C}$ to form the $\mathrm{ABF}$ matrix $\mathbf{A}$.

1) Exhaustive search (ES) method: Let $\mathcal{A}$ be the set of all $N \times R$ matrices, whose columns are obtained from set $\mathcal{C}$, i.e.,

$$
\mathcal{A}=\left\{\mathbf{A} \in \mathbb{C}^{N \times R} \mid \mathbf{a}_{r} \in \mathcal{C}, r \in \mathcal{R}\right\} .
$$

The cardinality of set $\mathcal{A}$, denoted by $V=|\mathcal{A}|=\frac{L !}{(L-R) ! R !}$. In the ES method we compute for each $\mathrm{ABF}$ matrix $\mathbf{A}_{v} \in \mathcal{A}$ the corresponding optimal DBF $\mathbf{b}_{v}$ by solving problem (5) and the corresponding transmit power $P_{v}=\left\|\mathbf{A}_{v} \mathbf{b}_{v}\right\|^{2}$, for $v \in \mathcal{V}=\{1, \ldots, V\}$. Subsequently, the optimal ABF matrix $\mathbf{A}_{v^{\star}}$ and the DBF $\mathbf{b}_{v^{\star}}$ that result in the smallest transmit power $P_{v}^{\star}$ are selected.

Even though the ES method yields the optimal solution for problem (3), its computational complexity grows exponentially with the codebook-size $L$ and the number of RF chains $R$.
Moreover, both the ABFs and the DBFs are computed at each symbol interval. Therefore this method becomes impractical even for a moderately large system.

2) Margin widening and selection operator (MWASO): In this method, the objective is to jointly perform the following two tasks: 1) maximize the minimum margin between the received signals and the decision boundaries of the corresponding transmit symbols among all users, and 2) minimize the number of selected codes. The problem is formulated as a sparse regularized feasibility problem, given by

$$
\begin{aligned}
& \underset{\delta \in \mathbb{R}, \overline{\mathbf{b}} \in \mathbb{C}^{L}}{\operatorname{minimize}} \delta+\epsilon|| \overline{\mathbf{b}} \|_{1} \\
& \text { s.t. }\left|\operatorname{Im}\left(\mathbf{h}_{k}^{\top} \mathbf{C} \overline{\mathbf{b}}\right)\right|-\left(\operatorname{Re}\left(\mathbf{h}_{k}^{\top} \mathbf{C} \overline{\mathbf{b}}\right)-\gamma_{k}\right) \tan \theta \leq \delta, \\
& \forall k \in \mathcal{K} .
\end{aligned}
$$

In the above problem, the real variable $\delta$ controls the minimum margin between the received signals and the corresponding decision boundaries of the transmit symbols among all users. The $\ell_{1}$-norm in the second term promotes sparsity in the selection vector $\overline{\mathbf{b}}[25,26]$. Moreover, it also controls the total transmit power. The weighting factor $\epsilon$ is a positive scalar. By appropriately choosing a value for $\epsilon$ (e.g., using bisection method), the vector $\overline{\mathbf{b}}$ can be adjusted to have exactly $R$ nonzero elements. Note that, in the above problem the left hand side of constraint (8b), which represents the true margin, is bounded by $\delta$ instead of zero in order to enable the problem to achieve any desired sparsity on vector $\bar{b}$.

Subsequently, the columns of codebook matrix $\mathbf{C}$ that corresponds to the non-zero elements of $\bar{b}$ are selected as ABFs. We remark that in this method the ABFs are designed based on the effective channel $\mathbf{h}_{k}$, which incorporates the transmit symbols, and therefore the $\mathrm{ABF}$ matrix must be redesigned at every symbol interval.

3) Best matching code selection (BMCS) method: In this method, for each user the $\mathrm{ABF}$ from the codebook $\mathcal{C}$ that maximizes the inner product with its channel vector is selected. To avoid selecting the same ABF multiple times, the selected $\mathrm{ABF}$ is removed from the codebook before selecting an $\mathrm{ABF}$ for the next user. The method is summarized in Alg. 1. Similar to the CPC method, this method designs the ABF matrix independent of the transmit symbol vector $\mathbf{s}$, and hence the $\mathrm{ABF}$ matrix can be kept fixed as long as the channel remains constant.

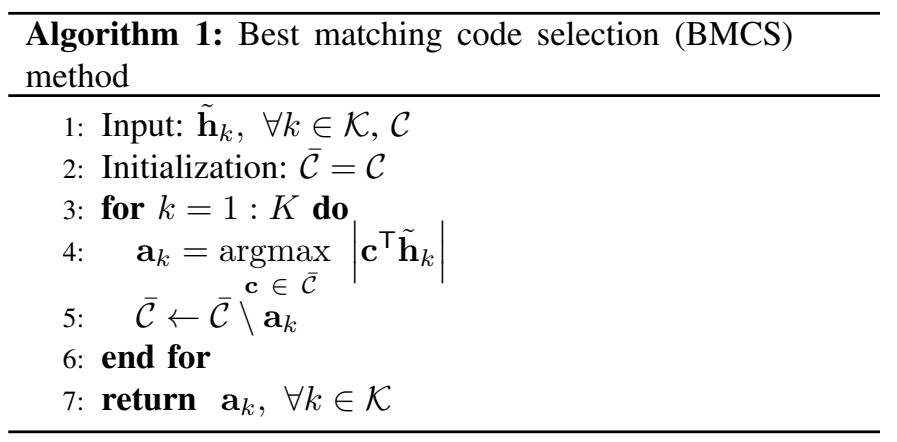




\section{NUMERICAL RESULTS}

In this section we compare the performance of all presented $\mathrm{ABF}$ design techniques. We also compare their performance with that of the conventional optimal fully-DBF ${ }^{1}$ and optimal antenna selection (OAS) $\operatorname{method}^{2}[9,10]$. For the simulation we employ the geometric channel model [24, 27]. The results are averaged over 5000 Monte-Carlo iterations.

Fig. 3 compares the transmit power required by the presented codebook-based ABF design techniques, when we employ a) the orthonormal DFT matrix based codebook, and b) the normalized random phase constant modulus codebook. We consider a system with $N=16$ transmit antennas, $K=2$ users, codebook-size $L=16$. In the figure we notice that the DFT codebook results in a lower transmit power when compared to the random codebook, for all three codebookbased ABF design techniques.

Fig. 4 compares the transmit powers (in $\mathrm{dB}$ ) of various $\mathrm{ABF}$ design techniques over a range of threshold-margin $\Gamma$ (at each user) for the above mentioned system. The DFT codebook is employed for the codebook-based ABF design methods. In the figure, we notice that the transmit power of the continuous-valued CPC method is very close to that of the optimal fully-DBF method in comparison with the transmit powers of the codebook-based ABF design methods. Table I shows that the computation time of the CPC method is lowest among all ABF techniques. From Fig. 4 and Table I we also infer that the codebook-based MWASO method achieves a performance close to that of the optimal ES method, with a significantly reduced computational complexity. The BMCS method is computationally very efficient when compared to the ES method and MWASO method, however, it results in substantially increased transmit power. Fig. 5 displays the transmit power of different $\mathrm{ABF}$ techniques ${ }^{3}$ for a larger system with $N=32$ transmit antennas over different number of users $K$. A $32 \times 32$ DBF codebook of is employed for MWASO and BMCS methods. The figure illustrates that the transmit power increase approximately linearly with the number of users $K$.

\section{CONCLUSION}

We have considered the multi-user downlink massive MIMO system with the BS equipped with hybrid analogdigital beamforming architecture, employing the CI based hybrid beamforming. The hybrid beamforming problem is decomposed into two parts: ABFs design and DBFs design. We have presented different $\mathrm{ABF}$ design techniques, and evaluated their performance in terms of required transmit power to meet a given quality of service requirement, as well as, computation time. We have also evaluated the effect of codebook selection on the performance of these techniques.

\footnotetext{
${ }^{1}$ When all $N$ antennas are employed and each antenna has a dedicated RF chain.

${ }^{2}$ For every subset of $R$ antennas, the optimal DBF is computed and then the subset of antennas that yields the smallest transmit power is selected.

${ }^{3}$ The ES and OAS methods become computationally impractical for this system-size, and hence they are excluded in the simulation.
}

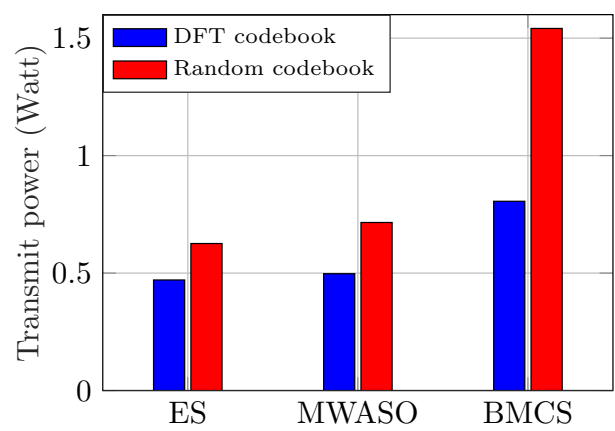

Fig. 3: Evaluation of effect of codebook selection on the performance of the ABF design techniques, for $N=L=16$, $R=K=2, M=4, \Gamma=0.7$.

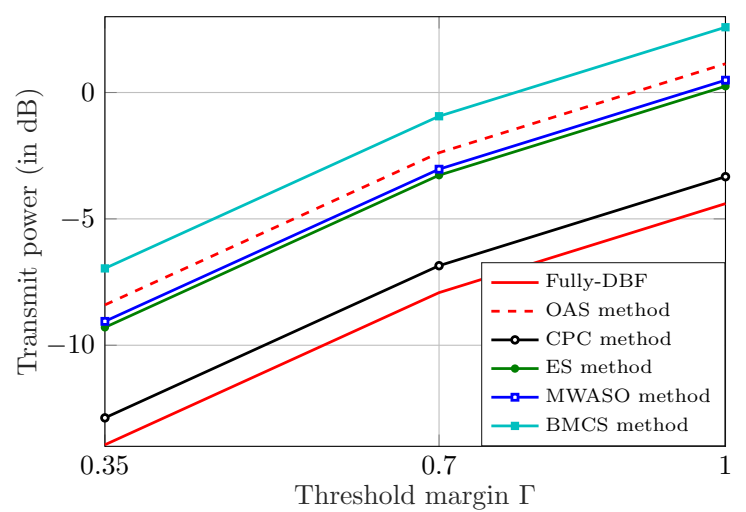

Fig. 4: Transmit power of various ABF design techniques for $N=L=16, R=K=2, M=4$.

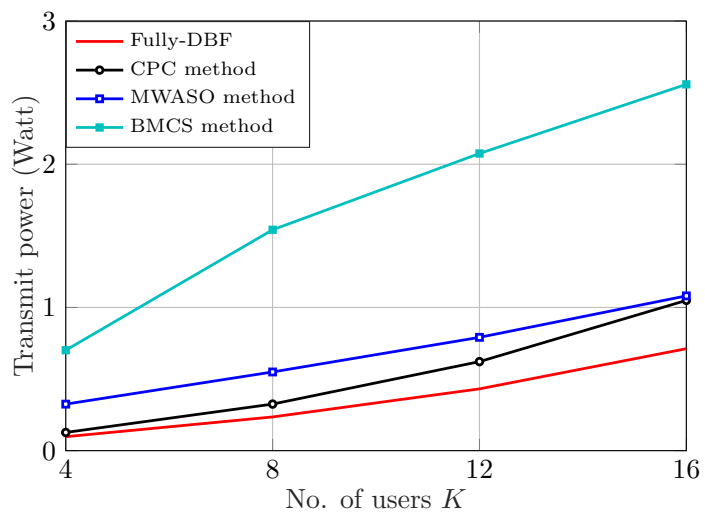

Fig. 5: Transmit power of various ABF design techniques for $N=L=32, M=4, \Gamma=0.7$, and $R=K$.

TABLE I: Computation time of different ABF design techniques for $N=L=16, K=2, M=4$.

\begin{tabular}{|l|c|}
\hline Method & Time (seconds) \\
\hline Fully-DBF & 0.30 \\
\hline OAS method & 21.27 \\
\hline CPC method & 0.23 \\
\hline ES method & 22.11 \\
\hline MWASO method & 5.19 \\
\hline BMCS method & 0.24 \\
\hline
\end{tabular}




\section{REFERENCES}

[1] W. Roh et al., "Millimeter-wave beamforming as an enabling technology for 5G cellular communications: Theoretical feasibility and prototype results," IEEE Commun. Mag., vol. 52, no. 2, pp. 106-113, Feb. 2014.

[2] O. E. Ayach, S. Rajagopal, S. Abu-Surra, Z. Pi, and R. W. Heath, "Spatially sparse precoding in millimeter wave MIMO systems," IEEE Trans. Wireless Commun., vol. 13, no. 3, pp. 1499-1513, Mar. 2014.

[3] T. E. Bogale and L. B. Le, "Beamforming for multiuser massive MIMO systems: Digital versus hybrid analogdigital," in Proc. IEEE Global Commun. Conf. (GLOBECOM), Austin, TX, USA, Dec. 2014, pp. 4066-4071.

[4] F. Sohrabi and W. Yu, "Hybrid digital and analog beamforming design for large-scale MIMO systems," in Proc. IEEE Int. Conf. on Acoustics, Speech and Signal Process. (ICASSP), Brisbane, Australia, Apr. 2015, pp. 29292933.

[5] G. Hegde, Y. Cheng, and M. Pesavento, "Hybrid beamforming for large-scale MIMO systems using uplinkdownlink duality," in Proc. IEEE Int. Conf. on Acoustics, Speech and Signal Process. (ICASSP), Mar. 2017, pp. 3484-3488.

[6] A. F. Molisch et al., "Hybrid beamforming for massive MIMO: A survey," IEEE Commun. Mag., vol. 55, no. 9, pp. 134-141, Sep. 2017.

[7] J. G. Andrews et al., "What will 5G be?" IEEE J. Select. Areas Commun., vol. 32, no. 6, pp. 1065-1082, Jun. 2014.

[8] F. Boccardi, R. W. Heath, A. Lozano, T. L. Marzetta, and P. Popovski, "Five disruptive technology directions for 5G," IEEE Commun. Mag., vol. 52, no. 2, pp. 74-80, Feb. 2014

[9] M. Schubert and H. Boche, "Iterative multiuser uplink and downlink beamforming under SINR constraints," IEEE Trans. Signal Process., vol. 53, no. 7, pp. 23242334, Jul. 2005.

[10] M. Bengtsson and B. Ottersten, "Optimal and suboptimal transmit beamforming," Handbook of Antennas in Wireless Commun., Aug. 2001.

[11] G. Zheng et al., "Rethinking the role of interference in wireless networks," IEEE Commun. Mag., vol. 52, no. 11, pp. 152-158, Nov. 2014.

[12] C. Masouros and G. Zheng, "Exploiting known interference as green signal power for downlink beamforming optimization," IEEE Trans. Signal Process., vol. 63, no. 14, pp. 3628-3640, Jul. 2015.

[13] M. Alodeh, S. Chatzinotas, and B. Ottersten, "Constructive multiuser interference in symbol level precoding for the MISO downlink channel," IEEE Trans. Signal Process., vol. 63, no. 9, pp. 2239-2252, May 2015.

[14] D. N. K. Jayakody, J. Thompson, S. Chatzinotas, and S. Durrani, Wireless Information and Power Transfer: A New Paradigm for Green Communications. Springer, 2017.
[15] S. Timotheou, G. Zheng, C. Masouros, and I. Krikidis, "Exploiting constructive interference for simultaneous wireless information and power transfer in multiuser downlink systems," IEEE J. Select. Areas Commun., vol. 34, no. 5, pp. 1772-1784, May 2016.

[16] A. Li and C. Masouros, "Hybrid analog-digital millimeter-wave MU-MIMO transmission with virtual path selection," IEEE Commun. Letters, vol. 21, no. 2, pp. 438-441, Feb. 2017.

[17] F. Rusek et al., "Scaling up MIMO: Opportunities and challenges with very large arrays," IEEE Signal Process. Mag., vol. 30, no. 1, pp. 40-60, Jan. 2013.

[18] H. Yin, D. Gesbert, M. Filippou, and Y. Liu, "A coordinated approach to channel estimation in large-scale multiple-antenna systems," IEEE J. Select. Areas Commun., vol. 31, no. 2, pp. 264-273, Feb. 2013.

[19] C. Masouros, T. Ratnarajah, M. Sellathurai, C. B. Papadias, and A. K. Shukla, "Known interference in the cellular downlink: A performance limiting factor or a source of green signal power?" IEEE Commun. Mag., vol. 51, no. 10 , pp. $162-171$, Oct. 2013.

[20] A. Li and C. Masouros, "Exploiting constructive mutual coupling in P2P MIMO by analog-digital phase alignment," IEEE Trans. Wireless Commun., vol. 16, no. 3, pp. 1948-1962, Mar. 2017.

[21] W. Ni and X. Dong, "Hybrid block diagonalization for massive multiuser MIMO systems," IEEE Trans. Commun., vol. 64, no. 1, pp. 201-211, Jan. 2016.

[22] A. Alkhateeb, G. Leus, and R. W. Heath, "Limited feedback hybrid precoding for multi-user millimeter wave systems," IEEE Trans. Wireless Commun., vol. 14, no. 11, pp. 6481-6494, Nov. 2015.

[23] A. Alkhateeb, O. E. Ayach, G. Leus, and R. W. Heath, "Channel estimation and hybrid precoding for millimeter wave cellular systems," IEEE J. Select. Topics in Signal Process., vol. 8, no. 5, pp. 831-846, Oct. 2014.

[24] L. Liang, W. Xu, and X. Dong, "Low-complexity hybrid precoding in massive multiuser MIMO systems," IEEE Wireless Commun. Letters, vol. 3, no. 6, pp. 653-656, Dec. 2014.

[25] E. J. Candes and M. B. Wakin, "An introduction to compressive sampling," IEEE Signal Process. Mag., vol. 25, no. 2, pp. 21-30, Mar. 2008.

[26] R. G. Baraniuk, "Compressive sensing [lecture notes]," IEEE Signal Process. Mag., vol. 24, no. 4, pp. 118-121, Jul. 2007.

[27] O. E. Ayach, S. Rajagopal, S. Abu-Surra, Z. Pi, and R. W. Heath, "Spatially sparse precoding in millimeter wave MIMO systems," IEEE Trans. Wireless Commun., vol. 13, no. 3, pp. 1499-1513, Mar. 2014. 